\title{
Synthesis and in vitro photobiological studies of porphyrin capped gold nanoparticles $\$$
}

\author{
ALBISH K PAUL $^{\mathrm{a}}$, DHANYA T JAYARAM ${ }^{\mathrm{a}}$, P S SANEESH BABU ${ }^{\mathrm{b}}$, \\ NAGAPPANPILLAI ADARSH ${ }^{\mathrm{a}}$, SHAMEEL THURAKKAL ${ }^{\mathrm{a}, \mathrm{c}}$, ASHA S NAIR $^{\mathrm{b}, *}$ and \\ DANABOYINA RAMAIAH ${ }^{\mathrm{d}, *}$ (]) \\ ${ }^{a}$ Photosciences and Photonics Section, Chemical Sciences and Technology Division, CSIR-National Institute \\ for Interdisciplinary Science and Technology, Thiruvananthapuram 695 019, Kerala, India \\ ${ }^{b}$ Rajiv Gandhi Centre for Biotechnology, Thiruvananthapuram 695 014, Kerala, India \\ ${ }^{c}$ Academy of Scientific and Innovative Research (AcSIR), CSIR-NIIST Campus, Thiruvananthapuram 695019 , \\ Kerala, India \\ ${ }^{\mathrm{d}}$ CSIR-North East Institute of Science and Technology, Jorhat 785 006, Assam, India \\ E-mail: rama@neist.res.in; d.ramaiah@gmail.com; sasha@ rgcb.res.in
}

MS received 26 May 2018; revised 13 July 2018; accepted 26 July 2018; published online 17 September 2018

\begin{abstract}
We describe the synthesis and characterization of a thiol-functionalized porphyrin derivative $\mathbf{2}$ and its gold nanoparticle conjugates. The porphyrin 2 exhibited its characteristic intense Soret absorption at $420 \mathrm{~nm}$ with a molar extinction coefficient value of $3.6 \times 10^{5} \mathrm{M}^{-1} \mathrm{~cm}^{-1}$ and good fluorescence in the region of 650$660 \mathrm{~nm}$. The porphyrin-capped gold nanoparticles (POPNPs) were synthesized from the citrate-capped gold nanoparticles by the ligand exchange method and characterized by spectroscopic and morphological analyses (UV-Vis, DLS and TEM). The broadening of the absorption spectrum and quenching of the fluorescence intensity for the porphyrin gold nanoconjugates suggest efficient incorporation of the porphyrin moiety onto the gold surface. The results of DLS and TEM analyses indicate that the nanoconjugate POPNPs are uniformly spherical in shape with a size of $c a .25 \pm 5 \mathrm{~nm}$ and exhibits a negative zeta potential value of $-16.0 \pm 2 \mathrm{mV}$. The singlet oxygen generation efficiency of the porphyrin 2 and POPNPs was calculated and are found to be $c a$. $0.53 \pm 0.02$ and $0.43 \pm 0.03$, respectively. The in vitro photobiological studies revealed that POPNPs exhibited enhanced photodynamic activity compared to their parent porphyrin derivative 2 with an $\mathrm{IC}_{50}$ value of $5 \mu \mathrm{M}$ in MDA MB 231 cell lines. The mechanism of the cell destruction was studied by Annexin-FITC and confirmed through TMRM assay. We observed the increase in the percentage of cell population corresponding to the late apoptotic stage $c a .37 .7 \%$ and $51.2 \%$ for 5 and $10 \mu \mathrm{M}$ of POPNPs, respectively, thereby demonstrating their apoptotic-mediated cell destruction and use in PDT applications.
\end{abstract}

Keywords. Gold nanoparticles; porphyrin; MTT assay; singlet oxygen; apoptosis.

\section{Introduction}

Photodynamic therapy (PDT) serves as an effective choice for the treatment of various cancers, which involves the combination of light, oxygen and a photosensitizing molecule. ${ }^{1-3}$ Strong absorption in the photodynamic window (600-800 nm), where the tissue penetration of light is maximum, high tumour specificity,

\footnotetext{
*For correspondence

$\S$ Dedicated to Professor M V George on the occasion of his $90^{\text {th }}$ Birth Anniversary.
}

efficient singlet oxygen generation, and negligible dark toxicity are the essential characteristics for an ideal photosensitizer. ${ }^{4}$ Several chromophores were investigated to study their photodynamic efficacies in different cell lines. ${ }^{5-7}$ Among those chromophores, the porphyrinbased molecules have been investigated extensively for PDT applications. These systems exhibit intense red fluorescence with good quantum yields of triplet excited state and singlet oxygen generation and excellent phototoxicity, thereby making them suitable candidates as photosensitizers for image guided therapy. ${ }^{8}$ 


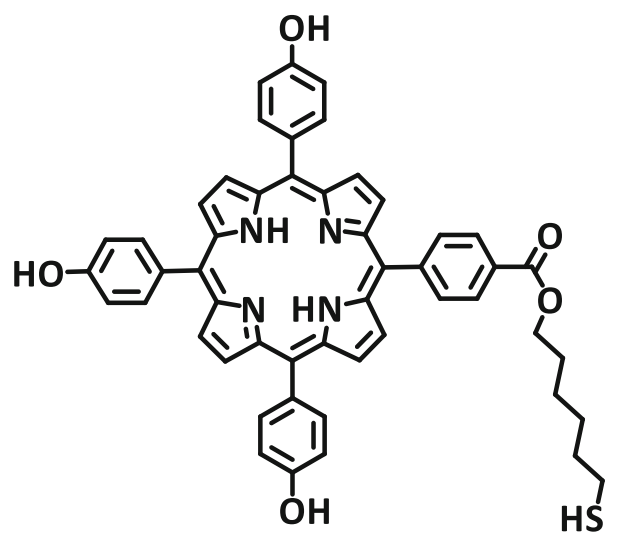

2

Chart 1. Structure of thiol-functionalized porphyrin 2.

Gold nanoparticles are emerging as promising agents for cancer therapy and are being investigated as drug carriers, photothermal agents, contrast agents and radiosensitizers. ${ }^{9}$ Nanoparticles may prevent the premature release of photosensitizers and they also have the advantage of enhanced permeability and retention for the improved cellular uptake. ${ }^{8}$ Most of the applications and studies of the functionalized nanoparticles involve the core modification as well as changing the nature of the stabilizing ligand. The core modification and change in size would enable one to tune the electronic and optical properties of the nanoparticles. However, the ligand exchange reactions have been proven to be effective approach for the incorporation of functionality in the ligands used in the thiol-capped nanoparticles and are widely used to produce both polar and non-polar nanoparticles. ${ }^{10-13}$

In this regard, we synthesized a thiol-functionalized porphyrin 2 (Chart 1) and have incorporated this photosensitizer onto the citrate-capped gold nanoparticles. Further, we have investigated the photobiological properties of the gold nanoparticles and found that the porphyrin-incorporated gold nanoparticles are more efficient in the cell destruction when compared to the bare gold nanoparticles as well as the parent porphyrin dye 2 .

\section{Experimental}

\subsection{Materials and methods}

The chemicals and reagents used in the study were purchased from SD Fine Chemicals, India; Sigma-Aldrich, USA; Merck Chemicals, Germany and were used as such without further purification. All experiments were carried out at room temperature $\left(25^{\circ} \mathrm{C} \pm 1{ }^{\circ} \mathrm{C}\right)$, unless otherwise mentioned. IR spectra were recorded on a PerkinElmer Model 882 infrared spectrometer. ${ }^{1} \mathrm{H}$ and ${ }^{13} \mathrm{C}$ NMR spectra were recorded on a $500 \mathrm{MHz}$ Bruker Avance DPX spectrometer. Electronic absorption spectra were recorded on a Shimadzu 2401 PC UV-Vis-NIR spectrophotometer. Fluorescence spectra were recorded on a PerkinElmer LS-55 spectrofluorimeter. Mass spectra were recorded on a Thermo Scientific Exactive ESIMS spectrophotometer. Fluorescence lifetimes were measured using an IBH (FluoroCube) picosecond time-correlated single photon counting (TCSPC) system. The samples were excited with a pulsed diode laser at $440 \mathrm{~nm}$ (NanoLED11) with a repetition rate of $1 \mathrm{MHz}$. The detection system consisted of a microchannel plate photomultiplier (5000U09B, Hamamatsu) with a 38.6 ps response time coupled to a monochromator $(5000 \mathrm{M})$ and TCSPC electronics [data station Hub including Hub-NL, NanoLED controller, and preinstalled fluorescence measurement and analysis studio (FMAS) software]. The fluorescence decay profiles were deconvoluted using IBH data station software V2.1 and minimizing the $\chi^{2}$ values of the fit to $1 \pm 0.1$. Fluorescence quantum yields were determined using tetraphenylporphyrin (TPP) as the reference compound ( $\Phi_{s}=0.11$ in toluene). ${ }^{14,15}$ Fluorescence quantum yields of the samples, $\Phi_{F}$ were calculated by Eq. 1,

$\Phi_{F}=\frac{\mathrm{A}_{s} \mathrm{~F}_{u} \mathrm{n}_{u}^{2}}{\mathrm{~A}_{u} \mathrm{~F}_{s} \mathrm{n}_{s}^{2}} \Phi_{s}$

wherein, $\mathrm{A}_{s}$ and $\mathrm{A}_{u}$ are the absorbance of standard and unknown, respectively. $\mathrm{F}_{s}$ and $\mathrm{F}_{u}$ are the integrated areas of fluorescence peaks of the standard and unknown and $\mathrm{n}_{s}$ and $\mathrm{n}_{u}$ are the refractive indices of the solvents used for the standard and unknown, respectively. $\Phi_{s}$ is the fluorescence quantum yield of the reference.

\subsection{Synthesis of 6-mercapto-hexan-1-ol}

6-Bromohexanol ( $1 \mathrm{~g}, 5.60 \mathrm{mmol})$ was allowed to reflux with thiourea $(0.64 \mathrm{~g}, 8.40 \mathrm{mmol})$ and $\mathrm{NaOH}(0.1 \mathrm{M})$ in $1,4-$ dioxane $(4 \mathrm{~mL})$ for $4 \mathrm{~h}$. The reaction mixture was cooled and the solvent was removed under vacuum. The $\mathrm{pH}$ of the reaction mixture was adjusted to 4 by adding dilute. $\mathrm{HCl}$ and the thiol derivative was extracted using dichloromethane. The organic layer was dried over $\mathrm{Na}_{2} \mathrm{SO}_{4}$ and concentrated to give 6-mercaptohexanol as an yellow oil.

Yield: $0.563 \mathrm{~g}(75 \%) ;{ }^{1} \mathrm{H}$ NMR $\left(500 \mathrm{MHz}, \mathrm{DMSO}_{-} \mathrm{d}_{6}\right.$, TMS): $\delta 3.65(\mathrm{~s}, 1 \mathrm{H}), 3.5(\mathrm{t}, 2 \mathrm{H}, J=2.4 \mathrm{~Hz}), 2.56(\mathrm{t}, 2 \mathrm{H}$, $J=2.3 \mathrm{~Hz}), 1.52-1.57(\mathrm{~m}, 4 \mathrm{H}), 1.5(\mathrm{~s}, 1 \mathrm{H}), 1.41-1.44(\mathrm{~m}$, $4 \mathrm{H}) ;{ }^{13} \mathrm{C}$ NMR (125 MHz, DMSO): $\delta 62.8,34.5,32.3,28.2$, 24.9, 24.6; ESI-MS: m/z Calcd. for $\mathrm{C}_{6} \mathrm{H}_{14} \mathrm{OS}: 134.08$, Found: $135.11\left(\mathrm{M}^{+}+1\right)$.

\subsection{Synthesis of the porphyrin 2}

Synthesis of porphyrin 2 involves the condensation of the porphyrin $\mathbf{1}$, which was prepared as per the reported procedure $^{16}$ with 6-mercaptohexan-1-ol in dry THF in presence of dicyclohexylcarbodiimide (DCC) and dimethyl 
<smiles>OCCCCCCBr</smiles>

6-bromohexan-1-ol
Thiourea, 1,4-Dioxane

$\mathrm{NaOH}, \mathrm{HCl}, 12 \mathrm{~h}$, $80^{\circ} \mathrm{C}$<smiles>OCCCCCCS</smiles>

6-Mercapto-hexan-1-ol

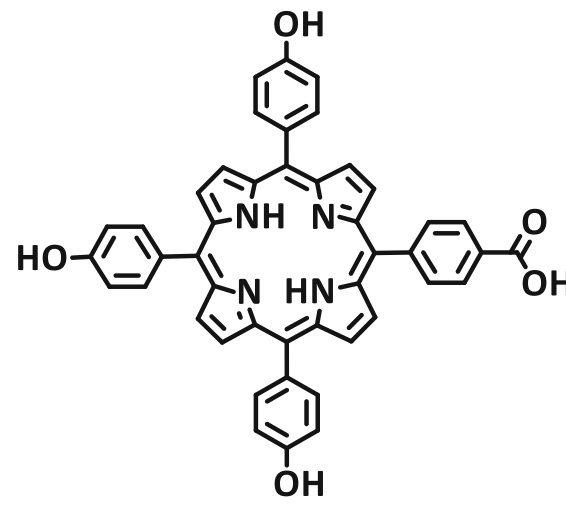

1

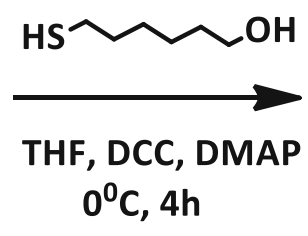

2

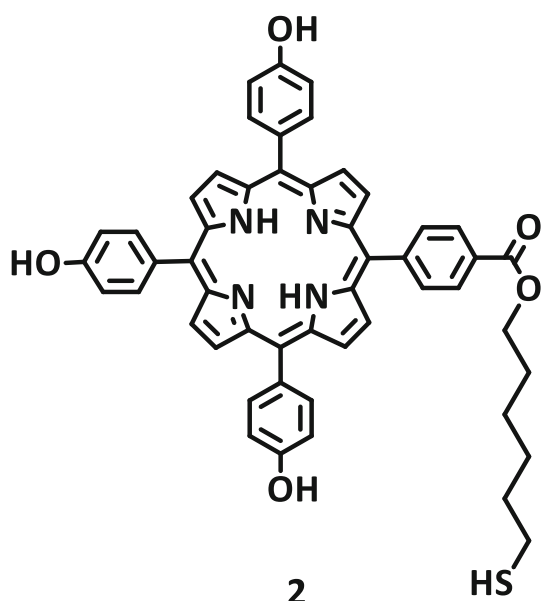

HS

Scheme 1. Synthesis of the thiol-functionalized porphyrin derivative 2 .

aminopyridine (DMAP). To an ice-cold solution of porphyrin 1 (2.26 g, $3.2 \mathrm{mmol})$ under argon atmosphere, DMAP (0.04 g, $0.32 \mathrm{mmol}$ ) was added and stirred for $15 \mathrm{~min}$. To this, DCC $(0.72 \mathrm{~g}, 3.5 \mathrm{mmol})$ was added followed by the 6mercaptohexan-1-ol $(0.43 \mathrm{~g}, 3.2 \mathrm{mmol})$ and stirred for $4 \mathrm{~h}$. The completion of the reaction was monitored by TLC. The reaction mixture was filtered, concentrated and washed with water and extracted using ethyl acetate. The organic layer was collected, dried over $\mathrm{Na}_{2} \mathrm{SO}_{4}$ and purified using column chromatography (5\% methanol-chloroform mixture).

Yield: $1.76 \mathrm{~g}(67 \%)$; M.p. $>300{ }^{\circ} \mathrm{C} ;{ }^{1} \mathrm{H}$ NMR $(500 \mathrm{MHz}$, DMSO-d $\left._{6}, \mathrm{TMS}\right): \delta 10.05(\mathrm{~s}, 3 \mathrm{H}), 8.93(\mathrm{~s}, 6 \mathrm{H}), 8.80(\mathrm{~s}$, $2 \mathrm{H}), 8.35-8.28(\mathrm{q}, 2 \mathrm{H}), 8.06-8.04(\mathrm{t}, 6 \mathrm{H}), 7.96-7.94(\mathrm{~d}, 2 \mathrm{H}$, $J=10), 7.26-7.24(\mathrm{t}, 6 \mathrm{H}), 4.45-4.40(\mathrm{t}, 1 \mathrm{H}), 2.02-2.00(\mathrm{~d}$, $2 \mathrm{H}), 1.49-1.44(\mathrm{t}, 2 \mathrm{H}), 1.34(\mathrm{~s}, 4 \mathrm{H}), 1.31(\mathrm{~s}, 4 \mathrm{H}),-2.85(\mathrm{~s}$, $2 \mathrm{H}$ ); ESI-MS: m/z Calcd. for $\mathrm{C}_{51} \mathrm{H}_{42} \mathrm{~N}_{4} \mathrm{O}_{5} \mathrm{~S}$ : 822.97, Found: $822.28\left(\mathrm{M}^{+}\right)$.

\section{Results and Discussion}

\subsection{Synthesis and characterization of the thiol-functionalized porphyrin 2}

The thiol-functionalized porphyrin $\mathbf{2}$ was synthesized by the coupling reaction between previously synthesized carboxyl porphyrin derivative $\mathbf{1}$ and 6-mercaptohexan1-ol using DCC and DMAP in THF as the solvent. The latter was obtained from the 6-bromohexan-1-ol by treating with thiourea in dioxane (Scheme 1). The starting materials as well as the products were characterized by spectroscopic and analytical methods such as IR, UV-Vis, NMR (Figure S1, Supplementary Information) and high-resolution mass spectrometry (HRMS).

\subsection{Synthesis and characterization of porphyrin capped gold nanoparticles (POPNPs)}

The basic methodology for the synthesis of porphyrin capped gold nanoparticles involved two steps: 1) preparation of the citrate-capped gold nanoparticles (AuNPs), and 2) ligand exchange of citrate-capped gold nanoparticles with the porphyrin derivative, 2 . The synthesis of citrate-capped gold nanoparticles involves the chemical reduction of the gold precursor $\mathrm{HAuCl}_{4}(0.25 \mathrm{mmol} / \mathrm{L})$ by dissolved trisodium citrate $(2.5 \mathrm{mmol} / \mathrm{L})$ at $75^{\circ} \mathrm{C}$ from their aqueous solutions. ${ }^{17-23}$ The yellow colored gold solution was turned into vine red, which indicates the formation of AuNPs and confirmed by the observation of the surface plasmon at $522 \mathrm{~nm}$ through UV-Vis spectroscopy (Figure S2, Supplementary Information). The ligand exchange between AuNPs and thiol porphyrin 2 was achieved by reacting AuNPs with the porphyrin derivative $\mathbf{2}$ in ethanol (Scheme 2). The ligand exchange reactions were carried out using different concentrations of the thiol porphyrin, $2(50 \mu \mathrm{M}, 100 \mu \mathrm{M}, 150 \mu \mathrm{M}, 200 \mu \mathrm{M}$ and $250 \mu \mathrm{M})$ to optimize the particle stability. Out of these 


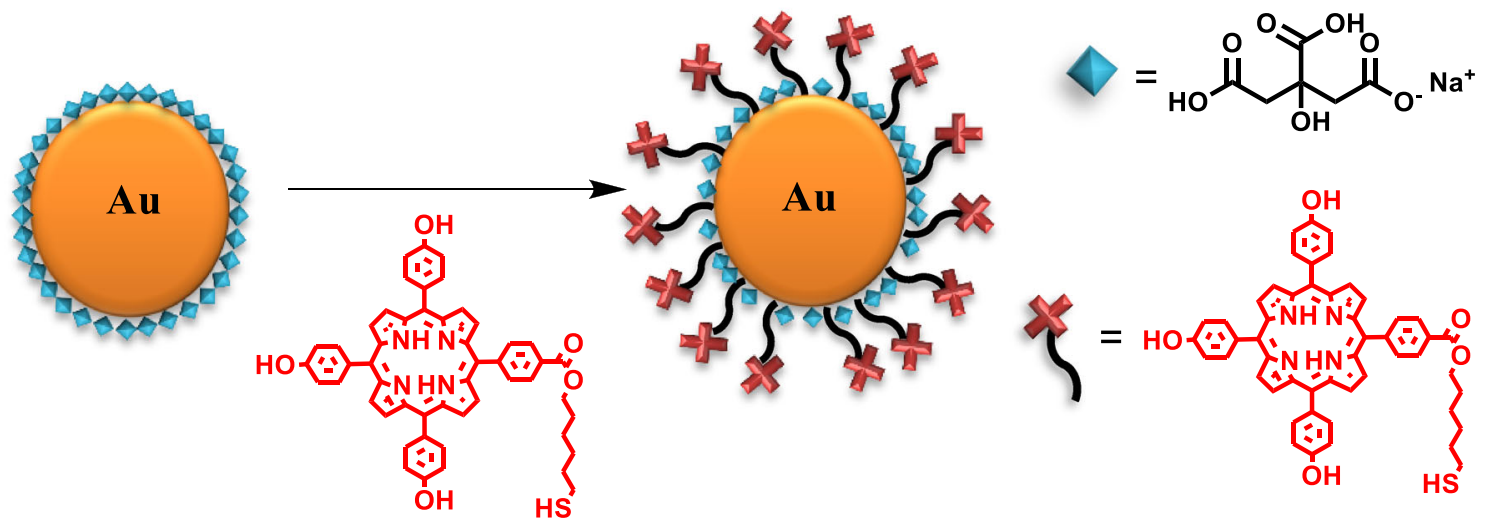

Scheme 2. Schematic representation of the synthesis of the porphyrin-capped gold nanoparticles (POPNPs) from the citrate-capped gold nanoparticles.
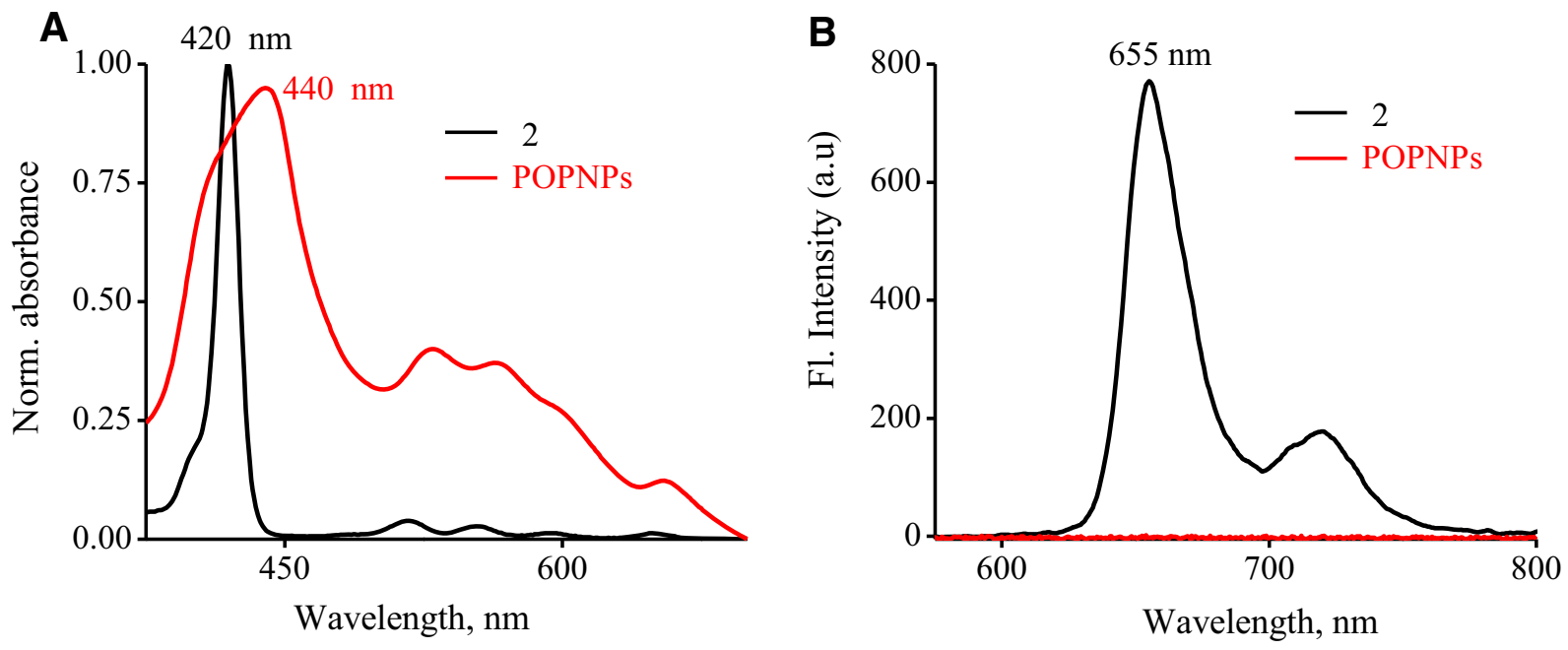

Figure 1. (A) Normalized absorption spectrum of the porphyrin $2(1.4 \mu \mathrm{M})$ in methanol (black trace) and the porphyrin-capped gold nanoparticles, POPNPs (red trace). (B) Fluorescence spectrum of the porphyrin 2 alone (1.4 $\mu \mathrm{M}$, black trace) and the POPNPs (red trace), excitation wavelength, $\lambda_{\mathrm{ex}}=515 \mathrm{~nm}$.

concentrations used, we obtained a maximum stability for the nanoparticles at a porphyrin concentration of $150 \mu \mathrm{M}$, which was evidenced through zeta potential measurements (Figure S3, Supplementary Information). The zeta potential value for the porphyrin bound gold nanoparticles (POPNPs) at $150 \mu \mathrm{M}$ of porphyrin, 2 was found to be $-6.0 \mathrm{mV}$. Hence, we selected this combination of gold nanoparticles for further studies. We observed a shift in the zeta potential value from $-40 \pm 2 \mathrm{mV}$ to $-16 \pm 2 \mathrm{mV}$ after the conjugation of the porphyrins onto the gold nanoparticles, which points towards the successful ligand exchange reaction and the citrate has been partially replaced by the porphyrin.

The thiol-functionalized porphyrin $\mathbf{2}$, showed the characteristic absorption and emission profiles of the porphyrin chromophore. It showed a Soret band in the absorption spectrum around $420 \mathrm{~nm}$ with extinction coefficient value of $3.6 \times 10^{5} \mathrm{M}^{-1} \mathrm{~cm}^{-1}$ and quinoid bands at 515, 552, 589 and $645 \mathrm{~nm}$ in methanol, as shown in Figure 1a. The absorption spectrum of the porphyrin-capped gold nanoparticles (POPNPs) was found to be broadened due to the electronic interactions of the gold (surface plasmon of the gold nanoparticles) with the porphyrin unit on the gold surface. Figure $1 \mathrm{~b}$ shows the fluorescence spectra of both the porphyrin and the porphyrin-capped gold nanoparticles. The fluorescence of the porphyrin 2 at $655 \mathrm{~nm}$ was quenched completely after ligand exchange with citrate capped AuNPs indicating the effective incorporation of the porphyrin moiety onto the gold surface. The fluorescence quantum yield of the porphyrin $\mathbf{2}$ was determined to be ca. $0.08 \pm 0.002$ using tetraphenylporphyrin (TPP) as the standard, ${ }^{14}$ whereas for the POPNPs, the value was found to be negligible. To have a better understanding of the fluorescence changes observed for the porphyrin as 
A

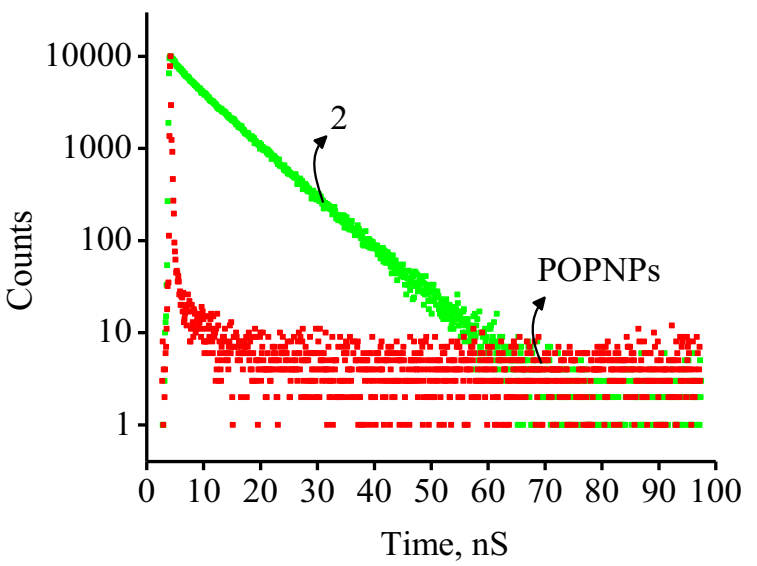

B

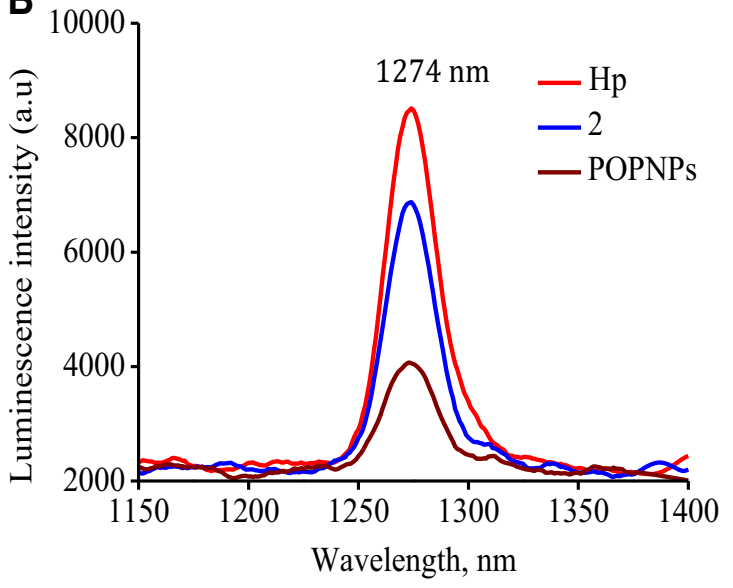

Figure 2. (A) Fluorescence decay profiles of the porphyrin 2 (green trace) and POPNPs (red trace). (B) Singlet oxygen luminescence at $1274 \mathrm{~nm}$ of Hematoporphyrin (Hp), porphyrin 2 and the nanoconjugate, POPNPs in acetonitrile (OD adjusted to 0.25 at excitation wavelength, $530 \mathrm{~nm}$ ).

A

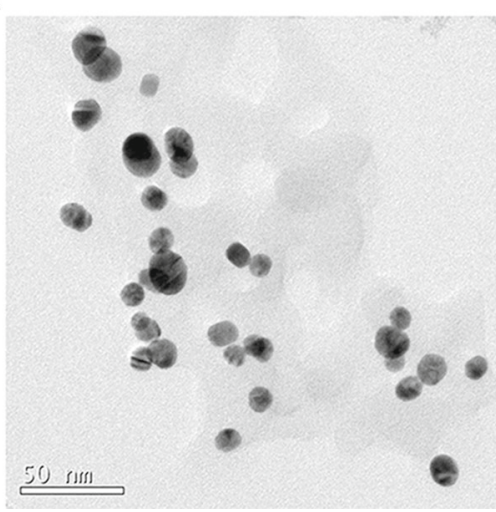

B

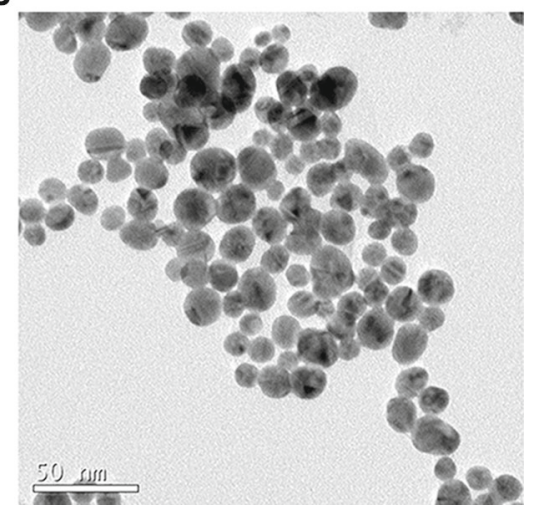

Figure 3. TEM images of (A) bare AuNPs and (B) POPNPs.

well as in the nanoparticle surface, we have analyzed the emission decay of the porphyrin through time-resolved fluorescence technique. The fluorescence decay profile of the porphyrin derivative $\mathbf{2}$ showed a monoexponential decay with a lifetime of $c a .8 .9$ ns. Notably, for the POPNPs, the fluorescence lifetime was found to be completely quenched (Figure 2a).

The efficient generation of singlet oxygen is a pre-requisite for a photosensitizing system to act as a potential PDT agent. In this context, we examined the efficiency of the thiol porphyrin, $\mathbf{2}$ as well as the nanoconstruct POPNPs through the direct method of monitoring the singlet oxygen luminescence at $1274 \mathrm{~nm}$ by excitation at $530 \mathrm{~nm}$ (Figure 2b). The quantum yield of singlet oxygen generation for the porphyrin, 2 and the nanoconjugate, POPNPs were calculated by using hematoporphyrin $(\mathrm{Hp})$ as the standard $\left(\Phi_{\Delta}=0.61 \pm 0.02\right)^{24}$ and the values are found to be $c a$. $0.53 \pm 0.02$ and $0.43 \pm 0.03$, respectively. The observed good quantum yield values for POPNPs suggest that the developed nanosystem can be effectively utilized for PDT applications.

To understand the morphology of the synthesized gold nanoparticles, we carried out dynamic light scattering (DLS) and transmission electron microscopy (TEM) experiments. ${ }^{25-27}$ In DLS analysis, we observed the formation of uniform spherical nanoparticles having a size of $c a .29 \pm 5 \mathrm{~nm}$ in aqueous media for the citrate capped nanoparticles (AuNPs) (Figure S4A, in Supplementary Information). In the case of POPNPs, we observed an increase in the size of the particle with an average size of $c a .47 \pm 5 \mathrm{~nm}$ indicating that the ligand exchange was effective (Figure S4B, Supplementary Information). We have further carried out the morphological analysis of the nanoparticles through TEM. TEM analysis of the citrate capped AuNPs (Figure 3a) confirmed that the nanoparticles were of spherical in shape with a size of ca. $20 \mathrm{~nm}$. Figure $3 \mathrm{~b}$ shows the corresponding TEM 

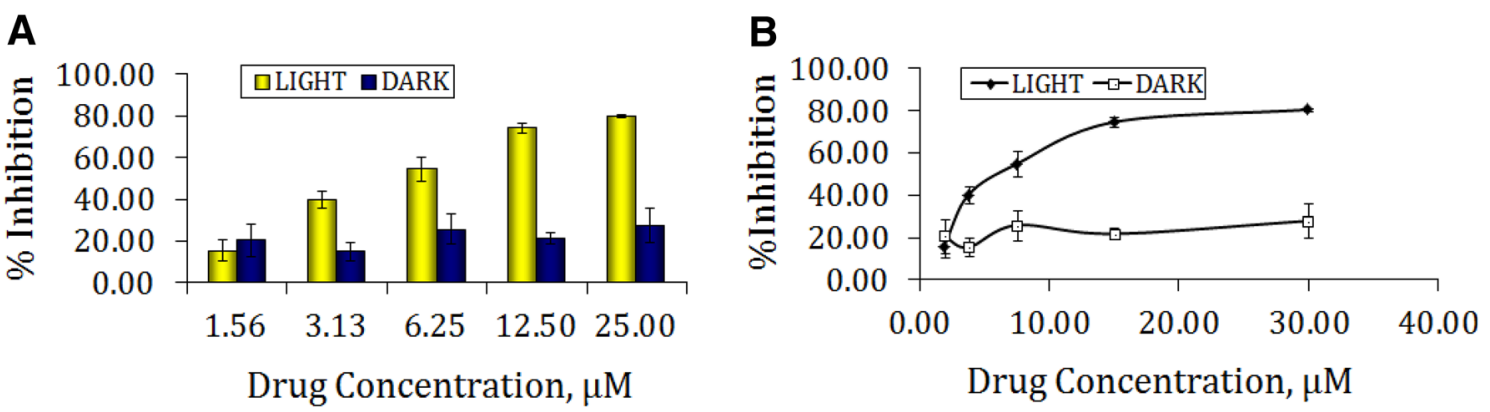

Figure 4. (A) Histogram showing the \% inhibition of porphyrin end-capped gold nanoparticles (POPNPs) in MDA MB 231 cells in light and dark conditions. (B) Concentration dependent cytotoxicity of POPNPs in dark and light conditions.

image of the POPNPs. Most of the particles were found to be spherical in shape with an average size of $c a$. $25 \mathrm{~nm}$. Further, the ${ }^{1} \mathrm{H}$ NMR spectrum of the POPNPs showed a peak broadening, which reveals that the thiol porphyrin is efficiently capped to the gold surface (Figure S5, Supplementary Information). In addition, the IR spectrum of the POPNPs got broadened as compared to the thiol porphyrin $\mathbf{2}$ indicating the interaction of the porphyrin derivative $\mathbf{2}$ with the gold surface (Figure S6, in Supplementary Information).

\subsection{In vitro photobiological activity of porphyrin-capped gold nanoparticles (POPNPs)}

As the porphyrin derivative $\mathbf{2}$ and POPNPs were quite efficient in generating singlet oxygen, we examined their in vitro photobiological activity under different conditions. To investigate the in vitro PDT of the POPNPs, we employed MTT assay in MDA MB 231 cell lines (aggressive human breast cancer) using $70 \mathrm{~W}$ Sodium vapor lamp $(\lambda>590 \mathrm{~nm}), 100 \mathrm{~J} \mathrm{~cm}^{-2}$. The half inhibitory concentration, $\mathrm{IC}_{50}$ value, was determined for the porphyrin dye as well as the POPNPs at dark and light conditions. The porphyrin derivative, $\mathbf{2}$ exhibited photocytotoxicity with an $\mathrm{IC}_{50}$ value of $25 \mu \mathrm{M}$. In contrast, we observed a significant enhancement in the photocytotoxicity for the porphyrin capped nanoparticles (POPNPs), which showed 5-fold better value of $\mathrm{IC}_{50}$ of $c a .5 \mu \mathrm{M}$ in MDA MB 231 cell lines (Figure 4). The photocytotoxicity of citrate-capped gold nanoparticles also evaluated as a control and which showed negligible cytotoxicity.

In apoptotic cells, the membrane phospholipid phosphatidylserine is translocated from the inner to outer leaflet of the plasma membrane, thus exposing phosphatidylserine to the external cellular environment. Annexin V (tagged with FITC) has high affinity for phosphatidylserine and therefore serves as a sensitive probe for identifying apoptotic cells by fluorescence microscopy and flow cytometry. ${ }^{28-30}$ To understand the mechanism behind the PDT activity and cellular damage induced by the porphyrin capped gold nanoparticles (POPNPs), we investigated Annexin V-FITC/PI assay using flow cytometric analysis (Figure 5). The cell populations at different phases, namely, viable (Annexin V-FITC-/PI-), early apoptotic (Annexin V-FITC+/PI-) and late apoptotic (Annexin V-FITC+/PI+) were examined at different sensitizer doses. We observed that most of the cells were viable for the light control (by irradiation in the absence of sensitizer) as well as for dark control (in presence of POPNPs $(10 \mu \mathrm{M})$ in the absence of light. The control irradiations were carried out by using the porphyrin 2 alone as well as citrate-capped gold nanoparticles alone and both of them showed negligible toxicity, which indicates that porphyrin derivative $\mathbf{2}$ as well as the gold nanoparticles are non-cytotoxic toward MDA-MB-231 cells. Interestingly, upon illumination of the porphyrin-capped gold nanoparticles (POPNPs), we observed the cell death through both early and late apoptosis in a concentration depended manner. The percentage of cell population corresponds to the necrosis/late apoptotic stage (Annexin V-FITC $+/ \mathrm{PI}+$ ) for $5 \mu \mathrm{M}$ of POPNPs was $c a .37 .7 \%$ and $10 \mu \mathrm{M}$ of POPNPs as $c a$. $51.2 \%$, whereas in light control and in presence of $\mathbf{2}$ alone, we observed only $c a$. $0.2 \%$ and $1.7 \%$, respectively (Figure 5). These results clearly indicate that the porphyrin-capped gold nanoparticles (POPNPs) induce cell death through late stage apoptosis.

To confirm the apoptotic mediated cell destruction, we have carried out tetramethylrhodaminemethyl ester (TMRM) staining experiment. The changes in mitochondrial membrane potential via apoptosis were monitored using the tetramethylrhodamine methyl ester through fluorescence imaging. The decrease in the mitochondrial inner membrane potential due to apoptosis, will results in the decrease in red fluorescence. We observed that upon, photodynamic treatment of MDAMB-231 cells with POPNPs at $5 \mu \mathrm{M}$ resulted in about 

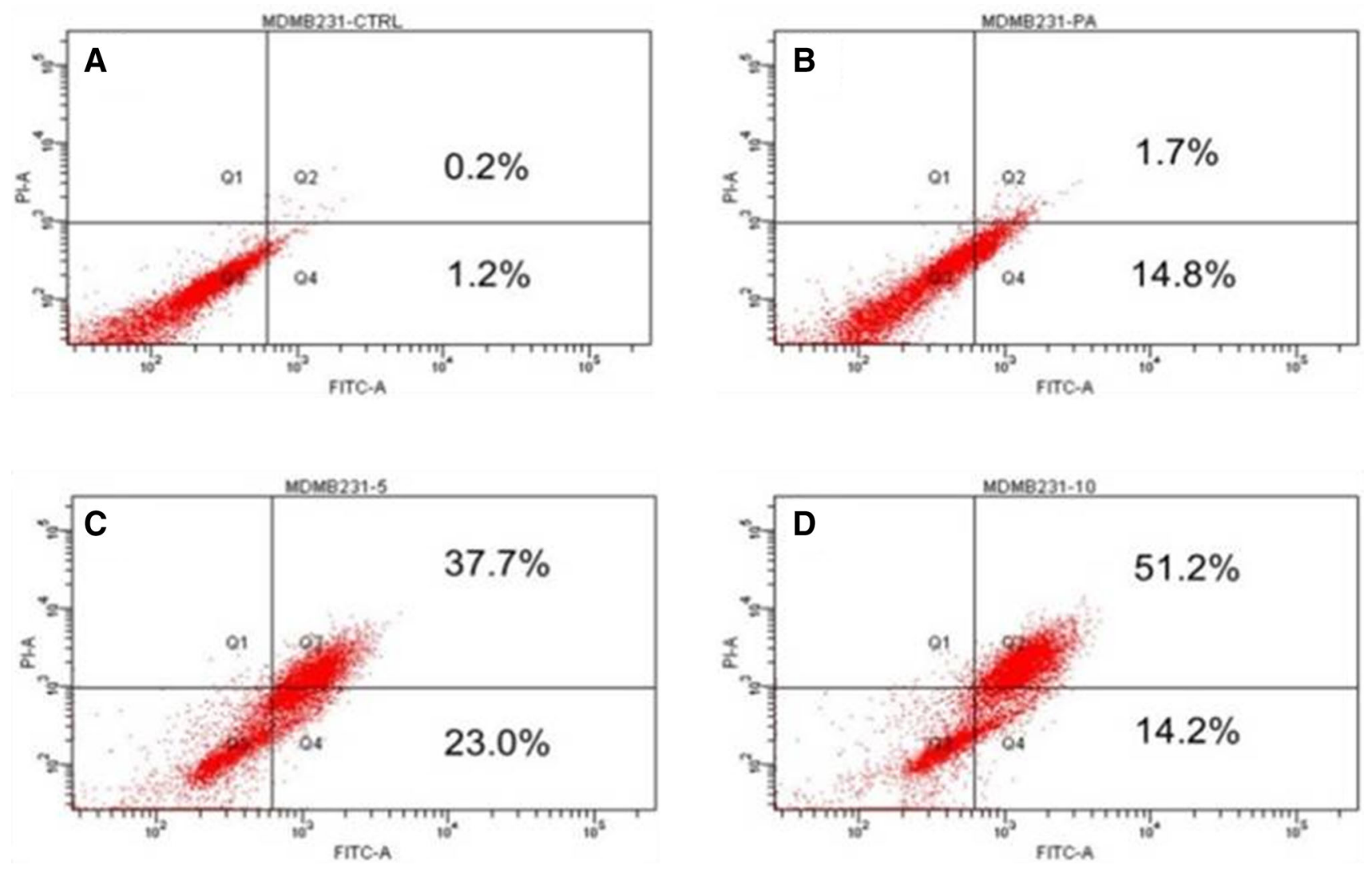

Figure 5. Flow cytometric analysis of MDA MB 231 cells after PDT treatment with POPNPs; (A) light control, (B) presence of 2 alone, (C) $5 \mu \mathrm{M}$ of POPNPs, and (D) $10 \mu \mathrm{M}$ of POPNPs.

A

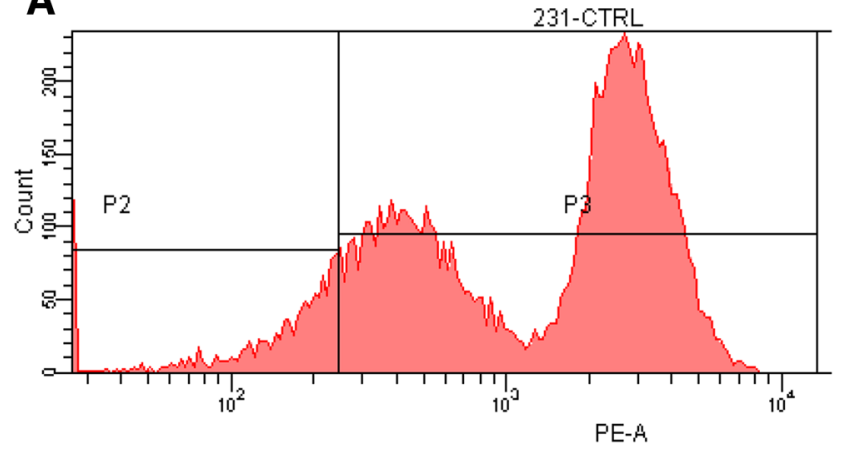

C

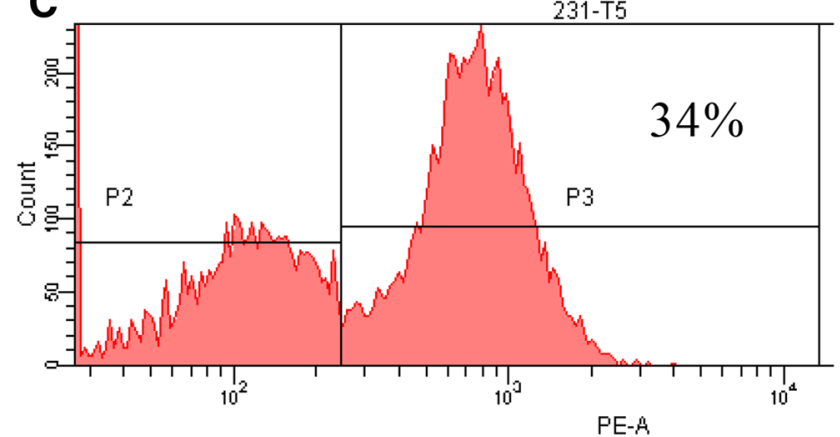

B

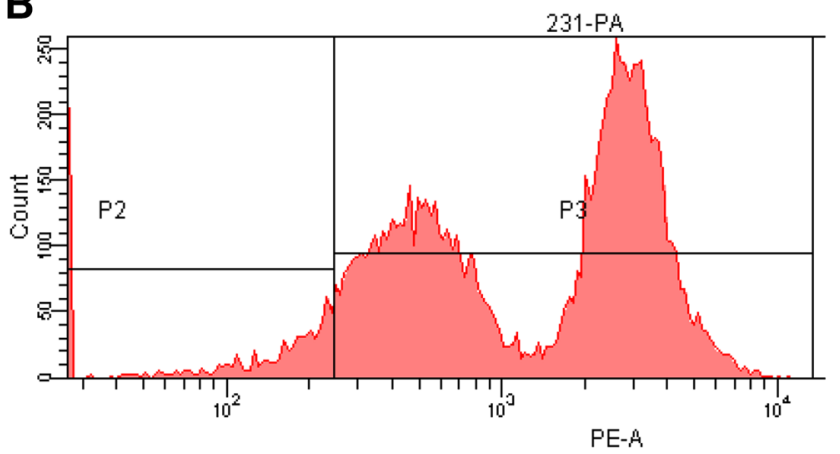

D

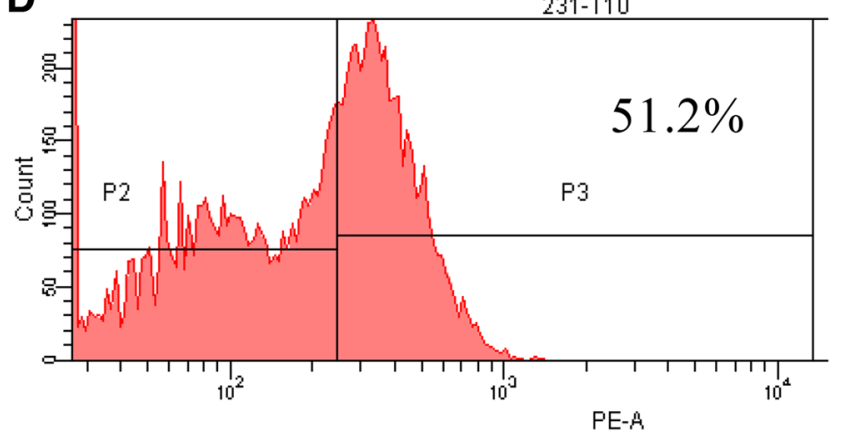

Figure 6. TMRM assay for the estimation of mitochondrial membrane potential reduction: Quantification through FACS analysis for (A) light control, (B) in presence of 2 alone, (C) $5 \mu \mathrm{M}$ of POPNPs, (D) $10 \mu \mathrm{M}$ of POPNPs. 
ca. $34 \%$ decrease in membrane potential, whereas at $10 \mu \mathrm{M}$ resulted in $c a .51 .2 \%$ decrease in mitochondrial membrane potential (Figure 6). On the other hand, the light and dark controls showed only $\mathrm{ca}$. $6 \%$ and $8 \%$ decreases in mitochondrial membrane potential. These observations confirm that the porphyrin-capped gold nanoparticles induce cell death during PDT treatment predominantly through apoptosis pathway.

\section{Conclusions}

In conclusion, we synthesized and characterized a thiol-functionalized porphyrin derivative $\mathbf{2}$ and investigated its photophysical as well as singlet oxygen generation properties. The good singlet oxygen generation efficiency of the thiol porphyrin led us to the incorporation of this porphyrin derivative in the gold nanoparticle surface by ligand exchange protocol. Various microscopic and photophysical studies have revealed that the thiol porphyrin was effectively incorporated into the gold nanoparticle surface (POPNPs). Further, we have carried out the cytotoxicity analysis of the gold nanoparticles by employing MTT assay and found that the porphyrin incorporated nanoparticles (POPNPs) were cytotoxic only in presence of light with an excellent $\mathrm{IC}_{50}$ value of $5 \mu \mathrm{M}$. The mechanism of the photodynamic action was found to be late apoptosis mediated cell destruction as confirmed by FITC-Annexin and TMRM assays. Our results demonstrate that porphyrin 2 can be used as an efficient sensitizer for the stabilization of gold nanoparticles thereby its effective use as a sensitizer in photodynamic therapeutic applications.

\section{Supplementary Information (SI)}

Supplementary Information for this article is available at www.ias.a.in/chemsci.

\section{References}

1. Ogawa K, Hasegawa H, Inaba Y, Kobuke Y, Inouye H, Kanemitsu Y, Kohno E, Hirano T, Ogura S and Okura I 2006 Water-Soluble Bis(imidazolylporphyrin) SelfAssemblies with Large Two-Photon Absorption Cross Sections as Potential Agents for Photodynamic Therapy J. Med. Chem. 492276

2. Ramaiah D, Joy A, Chandrasekhar N, Eldho N V, Das S and George M V 1997 Halogenated Squaraine Dyes as Potential Photochemotherapeutic Agents. Synthesis and Study of Photophysical Properties and Quantum Efficiencies of Singlet Oxygen Generation Photochem. Photobiol. 65783

3. Ramaiah D, Eckert I, Arun K T, Weidenfeller L and Epe B 2002 Squaraine Dyes for Photodynamic Therapy: Study of Their Cytotoxicity and Genotoxicity in Bacteria and Mammalian Cells Photochem. Photobiol. 76672

4. Zhang P, Steelant W, Kumar M and Scholfield M 2007 Versatile Photosensitizers for Photodynamic Therapy at Infrared Excitation J. Am. Chem. Soc. 1294526

5. Ethirajan M, Chen Y, Joshia P and Pandey R K 2011 The role of porphyrin chemistry in tumor imaging and photodynamic therapy Chem. Soc. Rev. 40340

6. Thomas A P, Saneesh Babu P S, Asha Nair S, Ramakrishnan S, Ramaiah D, Chandrashekar T K, Srinivasan A and Radhakrishna Pillai M 2012 meso-Tetrakis(psulfonatophenyl)N-Confused Porphyrin Tetrasodium Salt: A Potential Sensitizer for Photodynamic Therapy J. Med. Chem. 555110

7. Jayaram D T, Ramos-Romero S, Shankar B H, Garrido C, Rubio N, Sanchez-Cid L, Gómez S B, Blanco J and Ramaiah D 2016 In Vitro and in Vivo Demonstration of Photodynamic Activity and Cytoplasm Imaging through TPE Nanoparticles ACS Chem. Biol. 11104

8. Foote C S 1968 Mechanisms of Photosensitized Oxidation Science 162963

9. You Y, Gibson S L, Hilf R, Davies S R, Oseroff A R, Roy I, Ohulchanskyy T Y, Bergey E J and Detty M R 2003 Water Soluble, Core-Modified Porphyrins. 3. Synthesis, Photophysical Properties, and in Vitro Studies of Photosensitization, Uptake, and Localization with Carboxylic Acid-Substituted Derivatives J. Med. Chem. 463734

10. Lucky S S, Soo K C and Zhang Y 2015 Nanoparticles in Photodynamic Therapy Chem. Rev. 1151990

11. Dinkel R, Braunschweig B and Peukert W 2015 Fast and Slow Ligand Exchange at the Surface of Colloidal Gold Nanoparticles J. Phys. Chem. C 1201673

12. Merg A D, Zhou Y, Smith A M, Millstone J E and Rosi N L 2017 Ligand Exchange for Controlling the Surface Chemistry and Properties of Nanoparticle Superstructures ChemNanoMat 3745

13. Mineo P, Abbadessa A, Mazzaglia A, Gulino A and Scamporrino E 2017 Gold nanoparticles functionalized with PEGylate uncharged porphyrins Dyes Pigm. 141 225

14. Quimby D J and Longo F R 1975 Luminescence studies on several tetraarylporphins and their zinc derivatives $J$. Am. Chem. Soc. 975111

15. Paul A K, Karunakaran S C, Jayaram D T, Adarsh N, Joseph J and Ramaiah D 2016 Selective recognition of cyanide ions by amphiphilic porphyrins in aqueous medium J. Porphyr. Phthalocya. 201368

16. Paul A K, Karunakaran S C, Joseph J and Ramaiah D 2015 Amino Acid-Porphyrin Conjugates: Synthesis and Study of their Photophysical and Metal Ion Recognition Properties Photochem. Photobiol. 911348

17. Girgis O, Isabelle C, Michael J C and David A R 2012 Targeting the Oncofetal Thomsen-Friedenreich Disaccharide Using Jacalin-PEG Phthalocyanine Gold Nanoparticles for Photodynamic Cancer Therapy Angew. Chem. Int. Ed. $\mathbf{5 1} 6158$

18. Zhaowu Z, Jing J, Youqun L, Yanyan M, Jian W and Liping S 2010 Conjugating folic acid to gold nanoparticles through glutathione for targeting and detecting cancer cells Bioorg. Med. Chem. 185528

19. Shiao-Wen T, Jiunn-Woei L, Fu-Yin H, Yi-Yun C, MeiJhih L and Ming-His Y 2008 Surface-Modified Gold 
Nanoparticles with Folic Acid as Optical Probes for Cellular Imaging Sensors 86660

20. Gaiping L, Dan L, Lixue Z Z and Erkang W 2009 OneStep Synthesis of Folic Acid Protected Gold Nanoparticles and Their Receptor-Mediated Intracellular Uptake Chem. Eur. J. 159868

21. Jain S, Bch M B, Hirst D G and O'Sullivan J M 2014 Gold nanoparticles as novel agents for cancer therapy Brit. J. Radiol. 851010

22. Enustun B V and Turkevich J 1963 Coagulation of Colloidal Gold J. Am. Chem. Soc. 853317

23. Nair L V, Nazeer S S, Jayasree R S and Ajayaghosh A 2015 Fluorescence imaging assisted photodynamic therapy using photosensitizer-linked gold quantum clusters ACS Nano 95825

24. Marydasan B, Nair A K and Ramaiah D 2013 Optimization of Triplet Excited State and Singlet Oxygen Quantum Yields of Picolylamine-Porphyrin Conjugates through Zinc Insertion J. Phys. Chem. B 117 13515

25. Redmond R W and Gamlin J N 1999 A Compilation of Singlet Oxygen Yields from Biologically Relevant Molecules Photochem. Photobiol. 70 391

26. Wang $\mathrm{H}$ M, Jiang J Q, Xiao J H, Gao R L, Lin F Y and Liu X Y 2008 Porphyrin with amino acid moieties:
A tumor photosensitizer Chem.- Biol. Interact. 172 154

27. Ronn A M, Nbouri M, Abrahmson A L and Pecci L 1999 Evaluation of the Third Generation Photosensitiser SC102 in Two Animal Models Lasers Med. Sci. 14 307

28. Rovers J P, Saarnack A E, de Jode M, Sterenborg H J, Terpstra O T and Grahn M F 2007 Biodistribution and Bioactivity of Tetra-pegylated Metatetra(hydroxyphenyl)chlorin Compared to Native Metatetra(hydroxyphenyl)chlorin in a Rat Liver Tumor Model Photochem. Photobiol. 71211

29. Karunakaran S C, Saneesh Babu P S, Madhuri B, Marydasan B, Paul A K, Nair S A, Rao K S, Srinivasan A, Chandrashekar T K, Rao Ch M, Pillai R and Ramaiah D 2012 In Vitro Demonstration of Apoptosis Mediated Photodynamic Activity and NIR Nucleus Imaging through a Novel Porphyrin ACS Chem. Biol. 8 127

30. Prasanth C S, Karunakaran S C, Paul A K, Kussovski V, Mantareva V, Ramaiah D, Selvaraj L, Angelov I, Avramov L, Nandakumar $\mathrm{K}$ and Subhash N 2014 Antimicrobial Photodynamic Efficiency of Novel Cationic Porphyrins towards Periodontal Gram-positive and Gram-negative Pathogenic Bacteria Photochem. Photobiol. 90628 